\title{
Mapping continuous potentials to discrete forms
}

Chris Thomson, ${ }^{1}$ Leo Lue, ${ }^{2}$ and Marcus N. Bannerman ${ }^{1}$

1) School of Engineering, University of Aberdeen, Fraser Noble Building, Kings College, Aberdeen AB24 3UE, UK

2) Department of Chemical and Process Engineering, University of Strathclyde, James Weir Building, 75 Montrose Street, Glasgow G1 IXJ, $U K$

(Dated: 15 October 2013)

The optimal conversion of a continuous inter-particle potential to a discrete equivalent is considered here. Existing and novel algorithms are evaluated to determine the best technique for creating accurate discrete forms using the minimum number of discontinuities. This allows the event-driven molecular dynamics technique to be efficiently applied to the wide range of continuous force models available in the literature, and facilitates a direct comparison of event-driven and time-driven molecular dynamics. The performance of the proposed conversion techniques are evaluated through application to the Lennard-Jones model. A surprising linear dependence of the computational cost on the number of discontinuities is found, allowing accuracy to be traded for speed in a controlled manner. Excellent agreement is found for static and dynamic properties using a relatively low number of discontinuities. Molecular dynamics of the converted Lennard-Jones discrete potential outperforms traditional time-stepping methods for gases but is significantly slower at higher densities.

PACS numbers: Valid PACS appear here

Keywords: DEM, Event-Driven Dynamics, Stepped Potentials 


\section{INTRODUCTION}

Particle simulation techniques are now over 50 years old ${ }^{1}$ and have become a vital tool in exploring natural processes at all scales. Molecular dynamics, granular dynamics ${ }^{2}$, dissipative particle dynamics, and even smooth particle hydrodynamics ${ }^{3}$ algorithms are all fundamentally identical. They each attempt to solve classical equations of motion for a large number of particles which represent the mass of the system. In such models, conservative interactions between particles are typically defined through a pairwise additive inter-particle potential $\Phi(r)$, where $r$ is the distance between the particles. The force $\mathbf{F}_{i j}$ acting on particle $i$ due to particle $j$ is given by

$$
\mathbf{F}_{i j}=-\frac{\partial}{\partial \mathbf{r}_{i}} \Phi\left(\left|\mathbf{r}_{i}-\mathbf{r}_{j}\right|\right)
$$

where $\mathbf{r}_{i}$ is the position of particle $i$, and $\mathbf{r}_{j}$ is the position of particle $j$.

There are two broad categories of inter-particle potentials: continuous and discrete. For continuous potentials, the interaction energy is a continuous function of the particle positions. The Lennard-Jones potential is a classic example of a continuous potential:

$$
\Phi^{\mathrm{LJ}}(r)=4 \varepsilon\left[\left(\frac{\sigma}{r}\right)^{12}-\left(\frac{\sigma}{r}\right)^{6}\right]
$$

where $r$ is the distance between the two particles, $\varepsilon$ is the minimum interaction energy, and $\sigma$ is the separation distance corresponding to zero interaction energy.

Continuous potentials are prevalent in the simulation literature, beginning with the first simulations of Lennard-Jones systems by Verlet in $1967^{4}$ to the complex many-body potentials used for biological systems today ${ }^{5,6}$. One reason for their popularity may be due to the ease in which physical scaling laws can be implemented into the potential. For example, the $r^{-6}$ term in the Lennard-Jones potential was selected to match the known scaling of molecular dispersion forces. The finite difference techniques used in simulations of continuous potentials are also well-developed and are straightforward to implement ${ }^{7}$.

In discontinuous (also known as "stepped" or "terraced" 8 ) potentials, the interaction potential changes only at discrete locations. Discrete potentials are equally as popular as continuous potentials, due to their amenability to theoretical analysis, and are at the heart of thermodynamic perturbation theory $(\mathrm{TPT})^{9,10}$ and kinetic theory ${ }^{11}$. However, there has not been the same explosion of molecular force fields and software tools as for continuous 


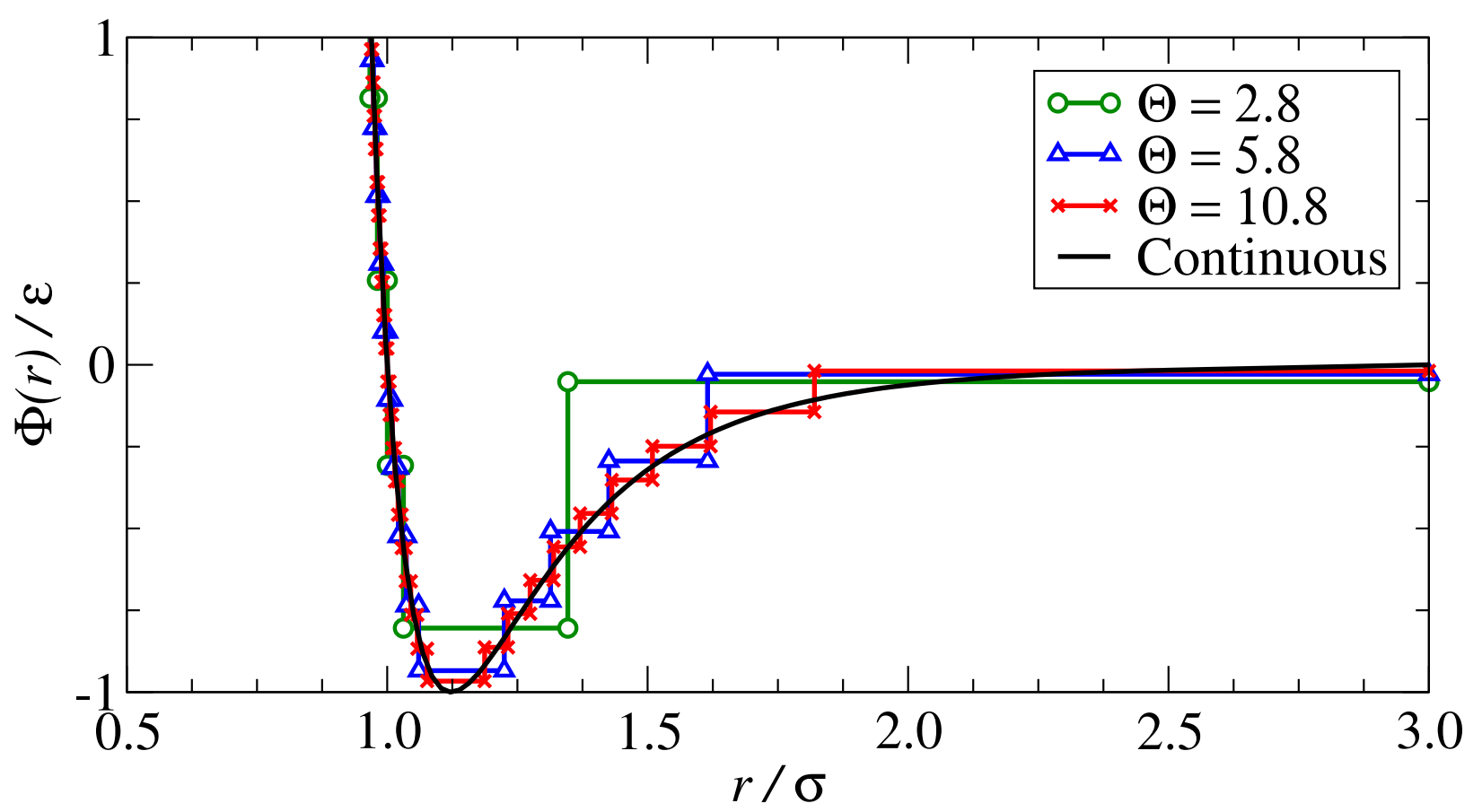

FIG. 1. A comparison of the continuous Lennard-Jones potential (solid) and three stepped approximations created using Eq. (4) for step placement and Eq. (11) for the step energies.

potentials (e.g., GROMACS ${ }^{12}$ and ESPResSo ${ }^{13}$ ). This is even more surprising given that the very first particle simulations were carried out using a discrete potential ${ }^{1}$, almost ten years before Verlet's simulations.

It is only relatively recently that fine-tuned discrete potentials for detailed, atomistic simulations have started to appear; these include force fields for a broad range of compounds, including hydrocarbons ${ }^{14}$ and fluorocarbons ${ }^{15}$, organic acids ${ }^{16}$, esters, ketones and other organic compounds ${ }^{17,18}$, phospholipids ${ }^{19}$, and peptides and proteins ${ }^{20}$. The use of TPT has even allowed rapid and direct fitting of discrete potentials to experimental data ${ }^{10,14,20,21}$. In addition, standard simulation packages for event-driven molecular dynamics have also begun to appear ${ }^{22}$.

The strong theoretical frameworks and stable simulation algorithm makes discrete potentials an attractive alternative to continuous potentials.

Complex discontinuous potentials are typically reported as a table of discontinuity locations and energies ${ }^{23}$. Although these two classes of potentials are distinct, it is clear that they may be made equivalent, provided a sufficient number of discontinuities or steps are used, as illustrated in Fig. 1. The optimal number, location, and energetic change of the dis- 
continuities for an accurate representation of a continuous potential is not well understood and is the subject of this paper.

It is desirable to have a mechanism to convert continuous potentials to discrete forms. This mapping must be optimized in the sense that it uses the smallest number of discontinuities to reduce the complexity of the converted potential and to minimize the computational cost of simulation. Chapela et al. ${ }^{23}$ was the first to attempt to represent the continuous Lennard-Jones potential by an equivalent discrete form. This mapping was optimized "by hand" to reproduce the thermodynamic properties at one state point, but more recent work $^{24-27}$ has focused on using regular stepping to automate the conversion process. Continuous potentials have also been used to directly specify the location of discontinuities ${ }^{8}$ allowing a convenient implementation of asymmetric potentials in event-driven dynamics; however, the optimization of this conversion is yet to be explored. Recently, there has been an attempt to replace the soft interactions of continuous potentials entirely with collision dynamics at low densities ${ }^{28}$ but this approach is restricted to low density systems.

In this work, the mapping of a continuous potential to a discrete form is investigated using the Lennard-Jones potential. In the following section, the placement of discontinuities and allocation of step energies is discussed before the methods are evaluated in Sec. III. The most efficient mapping scheme is then evaluated for a range of thermodynamic and transport properties in Sec. IV. A comparison between time-stepping and event-driven simulation is performed in Sec. V. Finally, the conclusions of the paper are presented in Sec. VI.

\section{DISCRETIZATION OF THE POTENTIAL}

The primary aim of this work is to develop an algorithm to convert a continuous potential to an optimal discrete form: one that provides an accurate approximation of the original continuous potential and can be simulated at a minimal computational cost. As the computational cost of an event-driven simulation is roughly proportional to the number of discontinuities encountered by the particles, it is vital that the number of discontinuities or steps used to achieve a set level of accuracy is minimized.

The location of a single step $i$ in a spherically-symmetric discrete potential is specified by the segment $\left[r_{i+1}, r_{i}\right]$ between the $i$ th and $i+1$ th discontinuities. The discontinuities, located at $r_{i}$ and $r_{i+1}$, bounding each step are also the limits of the neighboring steps. A 
simplification made in this work is to require that each step is directly representative of the segment of the continuous potential lying within the same limits $\left[r_{i+1}, r_{i}\right]$. This allows the task of discretizing the potential to be split into two smaller tasks: the optimal placement of discontinuities and the determination of an effective step energy for a segment of the continuous potential.

It is common to accelerate molecular dynamics calculations by truncating the interaction potential at a cut-off radius $r_{\text {cutoff }}$, thus requiring only local particle pairings to be considered in force calculations. Typically in time-stepping simulations, the potential is also shifted to eliminate the discontinuity at the cutoff in order to avoid the presence of impulsive forces. For example, the truncated, shifted Lennard-Jones potential is given by

$$
\Phi(r)=\left\{\begin{array}{ll}
\Phi^{L J}(r)-\Phi^{L J}\left(r_{\text {cutoff }}\right) & \text { if } r \leq r_{\text {cutoff }} \\
0 & \text { if } r>r_{\text {cutoff }}
\end{array} .\right.
$$

As each step of the discontinuous potential represents a segment of the original continuous potential, the first discontinuity is defined to lie at the cutoff radius (i.e. $r_{1}=r_{\text {cutoff }}$ ), while all other discontinuities lie within in the region $r \in\left(0, r_{\text {cutoff }}\right)$. It is tempting to also define an inner hard-core radius of the stepped potential using one of the available methods (e.g., see Ref. 29); however, this would require each step energy to somehow compensate for the overly repulsive core, inextricably linking step placement and energy once again. The available methods for placing discontinuities are reviewed in the next section before the algorithms used to generate representative step energies are discussed.

\section{A. Location of Discontinuities}

The simplest approach to place the discontinuities of a discrete potential is to divide the region $r \in\left(0, r_{\text {cutoff }}\right)$ into a number of steps of equal width $\Delta r^{25}$.

$$
r_{i, \Delta r}=r_{\text {cutoff }}-(i-1) \Delta r
$$

The total number of discontinuities/steps in the potential (including the cutoff) is given by $\left\lfloor r_{\text {cutoff }} / \Delta r\right\rfloor+1$.

It is not immediately clear that a uniform radial placement of the steps is the natural choice for a spherical potential. An alternative choice is to fix the volume $\Delta v$ bounded by 
each step of the potential. In this case, each step location is determined using the following recursive expression

$$
r_{i, \Delta v}=\left(r_{i-1, \Delta v}^{3}-\frac{3 \Delta v}{4 \pi}\right)^{1 / 3}
$$

The total number of discontinuities in the potential is then $\left\lfloor 4 \pi r_{\text {cutoff }}^{3} / 3 \Delta v\right\rfloor+1$.

The primary disadvantage of the approaches outlined above is that they do not attempt to adapt the step locations according to the behavior of the potential. It is likely that the performance of both algorithms is particularly sensitive to the configuration of the steps near the minimum of the potential where the interaction energy changes rapidly.

It has also been proposed ${ }^{8,27}$ to discretize continuous potentials by placing discontinuities at fixed intervals of interaction energy $\Delta \Phi$. This approach allows a controlled resolution of the potential, while balancing the contribution of each step and allows a straightforward extension to asymmetric potentials. The locations of the discontinuities are the ordered solutions to the following set of equations

$$
\Phi(r)=j \Delta \Phi \quad j \in \mathbb{Z}
$$

The application of Eq. (4) to the shifted, truncated Lennard-Jones potential results in an infinite number of steps due to the singularity at $r=0$. In practice, the high-energy steps are inaccessible and only a small number need to be computed during the simulation.

Before these approaches can be evaluated, a technique for determining the step energies must be selected. This is discussed in the following section.

\section{B. Step Energy}

With the location of each step defined through one of the above algorithms, an algorithm for determining the effective energy of a segment of the potential is required. In the limit of a large number of discontinuities/small segments, the original continuous potential must be

recovered. Chapela et al. ${ }^{25}$ have evaluated three approaches based on point sampling of the continuous potential.

$$
\begin{aligned}
\Phi_{i}^{\text {Left }} & =\Phi\left(r_{i+1}\right) \\
\Phi_{i}^{\text {Mid }} & =\Phi\left(\frac{r_{i}+r_{i+1}}{2}\right) \\
\Phi_{i}^{\text {Right }} & =\Phi\left(r_{i}\right),
\end{aligned}
$$


where $\Phi_{i}$ is the energy of step $i$ over the region $\left[r_{i+1}, r_{i}\right]$ of the discontinuous potential. Chapela et al. ${ }^{25}$ report that mid-point sampling $\left(\Phi_{i}^{M i d}\right)$ of the underlying continuous potential is the most effective at reproducing the original behavior of the Lennard-Jones potential, whereas left sampling is more appropriate for the Yukawa potential. It is easy to define other methods of point sampling, such as the minimum edge energy used by van Zon and Schofield $^{8}$. A straightforward choice is to sample the potential at the distance which divides the step into two equal volumes.

$$
\Phi_{i}^{M i d V o l}=\Phi\left(\left[\frac{r_{i}^{3}+r_{i+1}^{3}}{2}\right]^{1 / 3}\right) .
$$

It is also possible to define alternative approaches which do not rely on point-sampling, such as an equal area approach ${ }^{27}$; however, it is more desirable to directly match the thermodynamic properties of the converted potentials. Unfortunately, matching properties, such as the pressure, would require the use of an accurate free energy, which is typically unavailable. Successful attempts have been made to adjust stepped potentials to directly match the predictions of the TPT to experimental data for a range of thermodynamic properties ${ }^{26}$; however, the resulting equation of state is still approximate and the expressions are rather complicated. In this work, the focus is on directly reproducing the properties of the continuous potential system. One simple approach is to use the lowest order density correction to both the pressure and free-energy, given by the second virial coefficient, which is directly calculated from the interaction potential. The contribution of a segment of the potential to the second virial may be calculated as follows

$$
B_{2}\left(r_{i}, r_{i+1}, T\right)=-2 \pi \int_{r_{i+1}}^{r_{i}}\left(e^{-\beta \Phi(r)}-1\right) r^{2} \mathrm{~d} r
$$

where $\beta=1 /\left(k_{B} T\right), T$ is the absolute temperature of the system, and $k_{B}$ is the Boltzmann constant. The energy of the step can then be set to match the contribution to the second virial coefficient for the corresponding segment of the continuous potential, using the following expression

$$
\Phi_{i}^{\text {virial }}(T)=-k_{B} T \ln \left(\frac{3}{r_{i}^{3}-r_{i+1}^{3}} \int_{r_{i+1}}^{r_{i}} r^{2} e^{-\beta \Phi(r)} \mathrm{d} r\right) .
$$

Application of this algorithm leads to excellent agreement at low densities for the pressure; however, the algorithm has a cumbersome dependence on the temperature, which may not be available a priori (e.g., in the microcanonical ensemble). In the high-temperature limit, 
equating the virial contribution reduces to taking a volume average of the energy within a step:

$$
\Phi_{i}^{\text {Volume }}=\frac{3}{r_{i}^{3}-r_{i+1}^{3}} \int_{r_{i+1}}^{r_{i}} \Phi(r) r^{2} \mathrm{~d} r .
$$

This indicates that the volume averaged approach will also yield a good reproduction of the pressure near the ideal gas limit of high-temperature and low-density. It should be noted that both virial and volume-averaging approaches will set an infinite energy for the innermost step of the Lennard-Jones potential due to the singularity at $r=0$. This can have a dramatic effect on the potential as the step placement algorithms in Eqs. (3) and (4) use a finite number of steps to represent the repulsive core.

\section{COMPARISON OF MAPPING PROCEDURES}

To compare the various methods for mapping potentials, molecular dynamics simulations of $N=1372$ discontinuous and continuous Lennard-Jones particles with $r_{\text {cutoff }}=3 \sigma$ were performed over a range of densities, $\rho=N / V$, and temperatures, $k_{B} T$. To collect thermodynamic properties, each simulation was run for $20\left(m \sigma^{2} / \varepsilon\right)^{1 / 2}$ for equilibration before five production runs of $30\left(m \sigma^{2} / \varepsilon\right)^{1 / 2}$ were used to collect averages and obtain estimates of the uncertainty. Dynamical properties were collected using three runs, each $10^{3}\left(m \sigma^{2} / \varepsilon\right)^{1 / 2}$ in duration. Averages are reported here with error bars corresponding to the standard deviation between runs. Simulations for the continuous truncated, shifted Lennard-Jones potential were performed using the ESPResSo ${ }^{13}$ package with a time step of $0.002\left(m \sigma^{2} / \varepsilon\right)^{1 / 2}$ and a Langevin thermostat with a friction parameter of $1\left(\varepsilon / m \sigma^{2}\right)^{1 / 2}$. Discrete potential simulations were performed using the DynamO ${ }^{22}$ package with an Andersen thermostat controlled to $5 \%$ of the overall events. During the collection of dynamical properties, the thermostat is disabled after the equilibration period and the temperature is monitored to ensure it remains within $2 \%$ of the set value.

The mapping procedures must be evaluated on a basis of accuracy as a function of computational cost. As event-driven simulators process events at a constant rate, the computational cost is proportional to the number of events that must be processed per unit of simulation time. Each additional discontinuity within the potential will generate additional events provided particle pairs can access it; therefore, the computational cost is dominated 
by the number of discontinuities in the well of the potential. A straightforward parameter for the order of approximation of the potential, $\Theta$, can then be defined for each step placement algorithm as follows:

$$
\Theta=1+ \begin{cases}\left(r_{\text {cutoff }}-r_{\text {min }}\right) / \Delta r & \text { for Eq. (2) } \\ 4 \pi\left(r_{\text {cutoff }}^{3}-r_{\text {min }}^{3}\right) / 3 \Delta v & \text { for Eq. (3) } \\ -\Phi\left(r_{\text {min }}\right) / \Delta \Phi & \text { for Eq. (4) }\end{cases}
$$

where $r_{\min }=2^{1 / 6} \sigma$ is the location of the minimum of the Lennard-Jones potential. The parameter $\Theta$ is continuous, and as $\Theta \rightarrow \infty$, the continuous Lennard-Jones potential is recovered. The integer part of $\Theta$ corresponds to the number of discontinuities in the attractive section of the potential and at whole integer values of $\Theta$, a discontinuity is placed at the minimum of the potential.

\section{A. Placement of the discontinuities}

The methods for placing the discontinuities of a discrete potential (Eq. (2)-(4)) are evaluated first. An example comparison of the calculated pressure and internal energy for a high-density super-critical state point using the mid-point sampling algorithm (Eq. (6)) to set the step energies is given in Fig. 2. A temperature of $k_{B} T / \varepsilon=1.3$ is used in these simulations as it is well-above the critical temperature $k_{B} T_{c} / \varepsilon \approx 1.15$ of the $r_{\text {cutoff }}=3 \sigma$ system $^{30}$ to avoid entering the two-phase region. It is clear from this comparison alone that the algorithm using fixed energy intervals $(\Delta \Phi$, Eq. (4)) is superior to the other approaches. The algorithm demonstrates rapid convergence to the result obtained from the continuous potential simulations once $\Theta>2$, and allows a controlled approximation of the continuous potential. The slight offset in the pressure at higher values of $\Theta$ is later shown to be an artifact of using the mid-point sampling method for the step energy. The radial placement algorithm (Eq. (2), $\Delta r$ ) appears to converge towards the continuous potential result for the internal energy but requires a large number of steps and its performance is erratic. The radial and volumetric placement algorithms are particularly sensitive to the order of approximation. For example, the volumetric algorithm is only accurate for the internal energy at integer values of $\Theta$ which correspond to a discontinuity placed at the minimum of the potential. This highlights the importance of a good approximation of the potential in this region at high densities. 


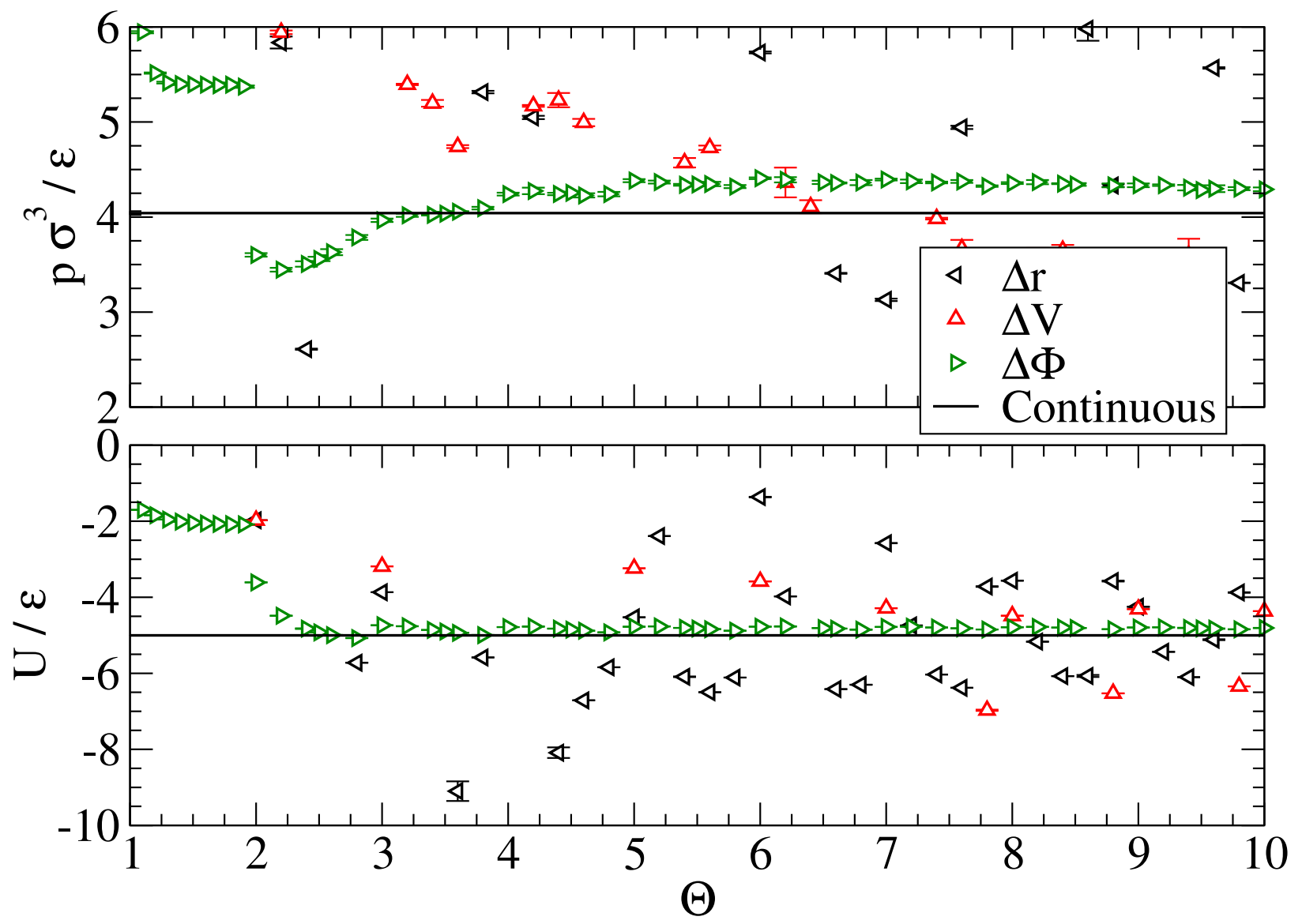

FIG. 2. Pressure, $p$, and internal energy, $U$, for a super-critical LJ liquid $\left(k_{B} T / \varepsilon=1.3, \rho \sigma^{3}=0.85\right)$ as a function of the number of attractive steps, $\Theta$, in the potential. The solid line indicates the continuous potential result. Each set of data points correspond to a different algorithm for placing the steps whereas the $\Phi_{i}^{\text {mid }}$ algorithm is used to set the step energy in all cases.

Further simulations have been carried out using all step-energy algorithms over a range of densities and temperatures and are in qualitative agreement with the trends outlined in Fig. 2; therefore, it is clear that the fixed energy interval algorithm given in Eq. (4) is the most appropriate as it is the only approach which allows a controlled approximation of the stepped potential. A full review of the available step energy algorithms using the fixed energy interval algorithm is performed in the following subsection. 


\section{B. Step energy}

The algorithms for setting the step energy, given in Eqs. (5)-(11), are evaluated by comparing predictions for the pressure (see Fig. 3) and internal energy (see Fig. 4) for two super-critical state-points at low and high density. Equation (4) is used to specify the step locations and the order of approximation is again controlled by specifying the number of discontinuities $\Theta$ in the attractive section of the potential. To confirm that $\Theta$ is the correct basis for comparison of these algorithms, the simulation event rates as a function of $\Theta$ are presented in Fig. 5. Each step-energy algorithm has an almost identical cost as a function of $\Theta$ and, for $\Theta>2$, a remarkable linear correlation is observed between the rate of events. As the event-driven simulation algorithm processes events at an approximately constant rate for a given cutoff and density, this demonstrates that there is a direct correspondence between $\Theta$ and the computational cost, making it a suitable basis for comparison.

At low densities, equating the virial contributions provides an excellent agreement for the pressure for all orders of approximation (see Fig. 3a), as expected. This is particularly interesting as for $\Theta \in(1,2)$ the discontinuous potential is a core-softened square-well potential. For predictions of the internal energy, the virial approach performs well only once a step is added between the minimum and the cutoff $(\Theta>2$ in Fig. 4a). The results of the Left sampling algorithm are not visible in the figures as they appear to enter the two-phase region, resulting in a very poor approximation. The Left, Mid, and Right algorithms display a strong dependence on the step placement through large changes near integer values of $\Theta$. The relatively smooth dependence of the Volume, and Virial algorithms on the $\Theta$ parameter indicates that these approaches provide a relatively unbiased sampling of the underlying potential. The MidVol algorithm performs worse overall than the Mid-point sampling approach and the Volume averaging algorithm performs almost as well as the Virial algorithm. The Volume algorithm appears to be a suitable replacement for the Virial if the temperature is not known and provided $\Theta>1$. At high densities (see Figs. 3b and 4b), the Virial and Volume averaging approaches still perform well but surprisingly the Mid-point sampling outperforms all other techniques for $1<\Theta<4$. This is likely due to a fortuitous cancellation of errors rather than an inherent advantage of the method, as its performance worsens for higher order approximations $(\Theta>4)$. It is clear that an excellent approximation is obtained for $\Theta>5$ with volume or virial stepping. 

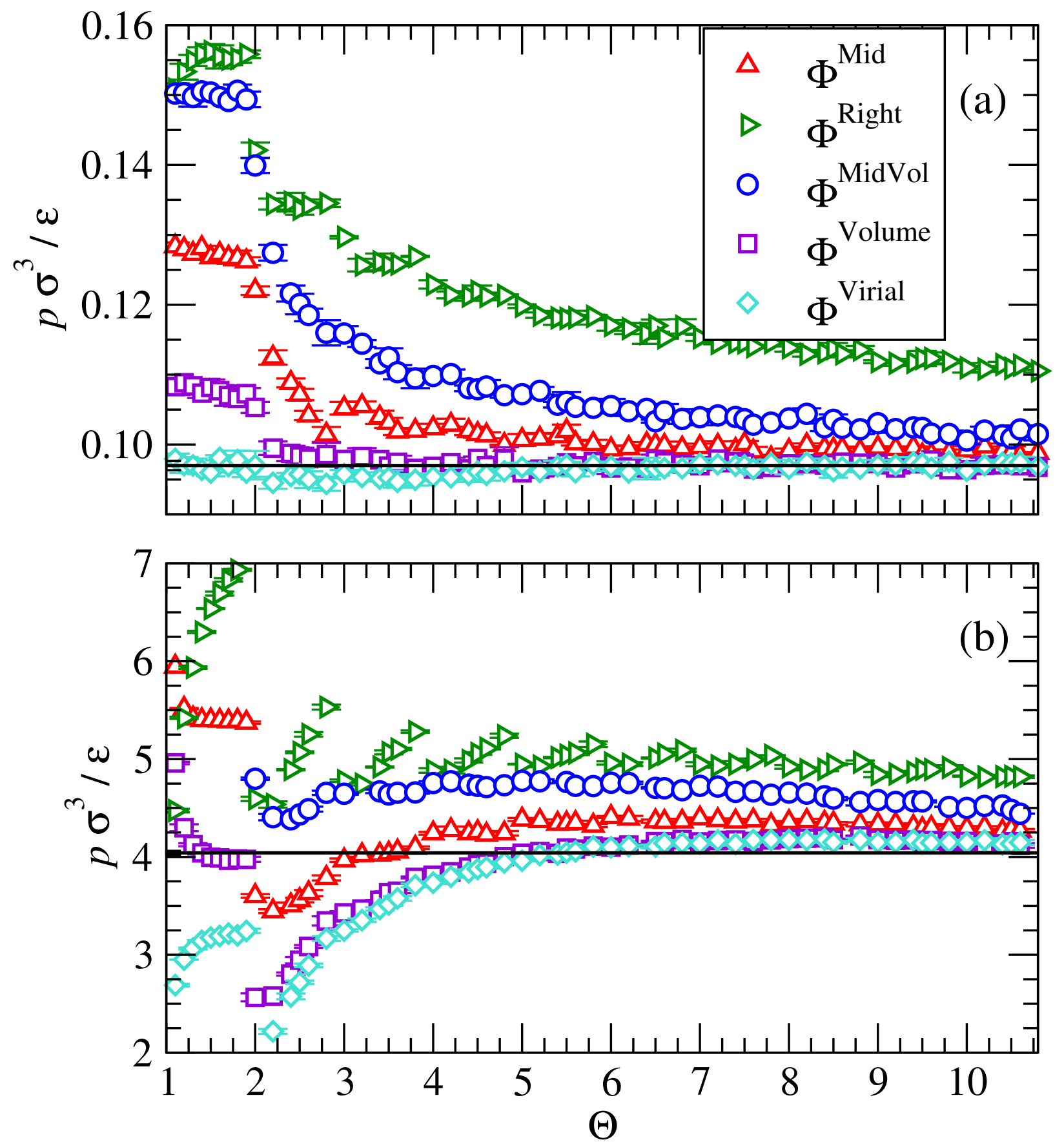

FIG. 3. A comparison of the stepped potential predictions for the pressure, $p$, of a $k_{B} T / \varepsilon=1.3$ Lennard-Jones fluid as a function of attractive step count $\Theta$ at (a) low $\left(\rho \sigma^{3}=0.1\right)$ and (b) high $\left(\rho \sigma^{3}=0.85\right)$ densities. The solid line indicates the continuous potential result and each symbol represents a different energy stepping algorithm (Eqs. (5)-(10)). Discontinuity locations are calculated using Eq. (4). Error bars are the standard deviations between the five production runs. 
Overall, setting the step energy through a volume average of the energy of the underlying continuous potential appears to yield a good approximation, provided $\Theta>1$. The additional complexity of a temperature-dependent potential through using the Virial approach does not appear justified at these conditions unless $\Theta<1$, but the reproduction of the internal energy is unacceptable at such a low order of approximation. Although it might be expected that the temperature dependence will become increasingly important at lower temperatures, further simulations carried out in the liquid branch at $k_{B} T / \varepsilon=0.85$ and $\rho \sigma^{3}=0.85$ yielded similar results to those reported, indicating the temperature correction is unjustified even when well within the liquid phase.

In summary, the placement of discontinuities using Eq. (4) and allocation of their energies using Eq. (11) appears to provide the best approximation of the continuous Lennard-Jones potential of the methods examined for the evaluated state points. As the computational cost primarily depends on the integer portion of $\Theta$ (see Fig. 5), it is optimal to select values of $\Theta$ with large fractional parts, such as $\Theta \approx 5.8$.

\section{OPTIMAL ALGORITHM EVALUATION}

The conversion algorithm which yielded the best performance, given in Eq. (4) and Eq. (11), is now fully evaluated across a wide range of state points. In particular, the trade-off between accuracy of reproduction and computational cost is explored. All values of $\Theta$ have a fractional part of .8 due to the step-wise scaling of the computational cost with $\Theta$ (see Fig. 5).

\section{A. Thermodynamic Properties}

To validate the thermodynamic properties of the system, the phase diagram (see Fig. 6) and vapor pressures (see Fig. 7) are calculated using Monte Carlo simulations in the grand canonical ensemble, using multi-canonical sampling to overcome the free energy barrier between the liquid and vapor phases ${ }^{31-33}$. The simulations were performed in a cubic box of side length $7 \sigma$. Approximately $100 \times 10^{6}$ configurations were sampled at each temperature, with 50\% attempted displacement moves and 50\% attempted particle insertion/deletion moves. Simulations were started near the critical point, and histogram re-weighting was 
used to determine the multi-canonical weights at the lower temperatures. The coexistence point at each temperature was determined by adjusting the chemical potential to equate the areas of the density histogram corresponding to the liquid and vapor phase.

The critical point is estimated by using a least-squares fit of the critical scaling of the density difference and the law of rectlinear diameters

$$
\begin{aligned}
\rho_{L}-\rho_{V} & =C_{1}\left(1-\frac{T}{T_{c}}\right)^{\beta_{c}} \\
\frac{1}{2}\left(\rho_{L}+\rho_{V}\right) & =\rho_{c}+C_{2}\left(T-T_{c}\right)
\end{aligned}
$$

where $\rho_{L}$ and $\rho_{V}$ are the liquid and vapor densities at a temperature of $T$, and $T_{c}$ and $\rho_{c}$ are the critical properties which, along with $C_{1}$ to $C_{2}$, are fitting parameters. A critical exponent of $\beta_{c}=0.3265$ is used here. The optimal conversion procedure appears to deliver a smooth convergence to the continuous potential result for the phase envelope in Fig. 6, highlighting the value of $\Theta$ as an order of approximation. The $\Theta=10.8$ system closely reproduces the thermodynamic behavior of the continuous Lennard-Jones potential, with only a slight under-estimation of the liquid transition density for low-temperature liquids. For values of $\Theta=3.8$ and 5.8, the approximation is remarkably close for such a low order approximation but for $\Theta=2.8$, the approximation rapidly deteriorates. Given the relatively low values of $\Theta$, the potential appears to be performing well when compared to previous conversions $^{23,25,27}$, although direct comparisons are difficult due to different choices for the cutoff radius. Results for the vapor pressures in Fig. 7 confirm the close reproduction of the continuous potential phase diagram for the $\Theta=10.8$ system.

Although the discrete approximations appear to converge to the thermodynamic properties of the Lennard-Jones fluid, there are subtle differences in the microscopic structure. The discontinuities in the energetic potential lead to discontinuities in $g(r)$, as illustrated for a high-density state point in Fig. 8. For low-order approximations the differences are significant, but for $\Theta=10.8$ the $g(r)$ is closely reproduced. The continuous cavity distribution function, defined as $y(r)=g(r) e^{\beta \Phi(r)}$, yields a close agreement between all approximations (see Fig. 8b). The sampling of $y(r)$ is poor for the continuous potential near $r \rightarrow 0.9$ as the $g(r)$ is low in this region; however, the use of the event-rate formulas allows a higher accuracy of sampling in this region for the stepped potential which explains the discrepancy. Despit these small differences in micro-structure, the overall thermodynamic properties of the system are effectively captured by the stepped potentials. 


\section{B. Dynamical Properties}

To fully validate the effectiveness of the conversion process, a number of dynamical properties have been calculated and are compared against the reference simulation results of Meier et al. ${ }^{35-37}$, and Bugel and Galliero ${ }^{38}$. Dynamical properties are difficult to calculate accurately as they require long simulation times to allow hydrodynamic behavior to appear. The literature values used here have been obtained using larger cut-offs (in the range of $r_{\text {cutoff }} / \sigma=5$ to 6 , depending on density). This will only cause small deviations except near the critical point of the literature fluid $\left(k_{B} T_{c} / \varepsilon \approx 1.34\right)$ which is above that of the $r_{\text {cutoff }} / \sigma=3$ fluid used here $\left(k_{B} T_{c} / \varepsilon \approx 1.17\right)$. Density sweeps are performed at two supercritical temperatures, $k_{B} T / \varepsilon=1.35$ near to the critical point of the reference data and $k_{B} T / \varepsilon=2.5$. Relative error estimates are obtained by combining the standard deviation between simulation runs for both the reference and discontinuous results.

Results for the self-diffusion coefficient are presented in Fig. 9. It is clear that dynamical properties such as the diffusion coefficient are much more difficult to approximate when compared with the thermodynamic properties. Low-order approximations have a reduced diffusion coefficient when compared to the continuous potential. This effect is due to the difference in critical temperatures which results in an lower reduced temperature for the low-order approximations in the theorem of corresponding-states. The results for $\Theta=10.8$ are acceptable with a maximum deviation of $\approx 8 \%$ over both isotherms.

Results for the viscosity are presented in Fig. 10. The $\Theta=10.8$ results are within $10 \%$ of the continuous results for both isotherms, except at the lowest two densities. The disagreement at low densities is likely due to the increased proportion of glancing interactions which shifts the emphasis from a close reproduction of the well to the outer tail of the potential. It may be that regular stepping of the potential using radial or volumetric placement is more suitable at very low gas densities. Overall, agreement is good given the uncertainty in the results.

Results for the thermal conductivity are presented in Fig. 11. Agreement is good for high temperatures but for the $k_{B} T / \varepsilon=1.35$ isotherm the stepped approximations significantly underestimate the thermal conductivity of the continuous potential. The disagreement at low-density may again be caused by an increase in glancing interactions but the discrepancy persists at moderate densities. Although there is typically very little change in the thermal 
conductivity with cutoff range there is an enhancement of the thermal conductivity in region of the critical point ${ }^{38}$. As the literature values at this temperature are near the critical point the stepped potential results will slightly under predict the thermal conductivity. For liquid densities $\left(\rho \sigma^{3} \gtrsim 0.6\right)$, the stepped approximation satisfactorily reproduces the continuous potential behavior.

\section{COMPUTATIONAL COST}

Using the selected conversion procedure, the computational cost of stepped/event-driven and continuous/time-stepping techniques can be compared on an equal basis. The relative speed of each method, defined as the simulation time processed per unit of CPU time, for

a $k_{B} T / \varepsilon=1.3$ isotherm is presented in Fig. 12. It is immediately apparent that the use of event-driven methods is only advantageous at gas densities $\left(\rho \sigma^{3} \lesssim 0.02\right)$ or below, where it significantly outperforms time stepping methods. Hybrid time-stepping/event-driven methods also display this dramatic increase in performance at low densities ${ }^{39}$ as the system dynamics becomes dominated by two-particle collisions. The use of stepped potentials will eliminate some of the overhead of hybrid techniques which indicates promising future applications of the potentials developed here in rarefied gas flow simulation. The comparison carried out here is only for serial execution performance; however, parallel algorithms for event-driven simulation exhibit good scaling ${ }^{40}$ and will be evaluated in future.

\section{CONCLUSIONS}

In this work, we have examined several methods for mapping a continuous interaction potential to a discrete, stepped potential. These methods were compared on their ability of the resulting discrete potential to reproduce the thermodynamic and transport properties of the continuous potential system over a broad range of conditions. Of the various methods which were examined to locate the discontinuities of the potential, the best was found to be at fixed intervals of energy. Setting the step energy through a volume average of the energy of the underlying continuous potential appears to give the a good overall approximation provided $\Theta>1$ and an excellent approximation for $\Theta=10.8$.

The computational cost for simulating the continuous potential and several levels of 
stepped potential approximation were compared on the basis of their processing rates for a unit of simulation time. Stepped Lennard-Jones potentials are increasingly efficient when compared to continuous forms at gas densities or lower $\left(\rho \sigma^{3} \lesssim 0.02\right.$ which is around $33 \mathrm{~kg} / \mathrm{m}^{3}$ for Argon). This indicates the potentials here may be used to significantly accelerate simulations of shock waves ${ }^{39}$ or other complex rarefied gas systems where DSMC techniques are currently applied.

Short range or repulsive potentials, such as the Weeks-Chandler-Anderson potential, should prove far more efficient targets for conversion given the speed of hard-sphere simulations. The Hertz potential, used in simulations of solids particles, is particularly interesting as the stepped equivalent may be arbitrarily steep. This, combined with the analytical dynamics of the event-driven technique, will allow the stable and efficient use of realistic materials parameters in granular simulations. The only obstacle to this application is the conversion of the dissipative inter-particle forces which will be explored in a future publication.

\section{ACKNOWLEDGMENTS}

The authors would like to acknowledge the support of the Maxwell compute cluster funded by the University of Aberdeen.

\section{REFERENCES}

${ }^{1}$ B. J. Alder and T. E. Wainwright, J. Chem. Phys. 27, 1208 (1957).

${ }^{2}$ T. Pöschel and T. Schwager, Computational Granular Dynamics (Springer, New York, 2005).

${ }^{3}$ R. A. Gingold and J. J. Monaghan, Mon. Not. R. Astron. Soc. 181, 375 (1977).

${ }^{4}$ L. Verlet, Phys. Rev. 159, 98 (1967).

${ }^{5}$ J. Ponder and D. Case, Adv. Prot. Chem. 66, 27 (2003).

${ }^{6}$ A. MacKerel Jr., C. Brooks III, L. Nilsson, B. Roux, Y. Won, and M. Karplus, in The Encyclopedia of Computational Chemistry, Vol. 1, edited by P. von R. Schleyer (John Wiley \& Sons: Chichester, 1998) pp. 271-277. 
${ }^{7}$ J. M. Haile, Molecular Dynamics Simulation - Elementary Methods (Wiley-Interscience, New York, 1997).

${ }^{8}$ R. van Zon and J. Schofield, J. Chem. Phys. 128, 154119 (2008).

${ }^{9}$ J. A. Barker and D. Henderson, J. Chem. Phys. 47, 2856 (1967).

${ }^{10}$ O. Unlu, N. H. Gray, Z. N. Gerek, and J. R. Elliott, Ind. Eng. Chem. Res. 43, 1788 (2004).

${ }^{11}$ S. Chapman and T. G. Cowling, The Mathematical Theory of Non-uniform Gases, 3rd ed. (Cambridge Mathematical Library, 1991).

${ }^{12}$ D. Van der Spoel, E. Lindahl, B. Hess, G. Groenhof, A. E. Mark, and H. J. C. Berendsen, J. Comput. Chem. 26, 1701 (2005).

${ }^{13}$ H.-J. Limbach, A. Arnold, B. A. Mann, and C. Holm, Comput. Phys. Commun. 174, 704 (2006).

${ }^{14}$ J. Cui and J. J. Richard Elliott, J. Chem. Phys. 116, 8625 (2002).

${ }^{15}$ A. D. Sans and J. R. Elliott, Fluid Phase Equilib. 263, 182 (2008).

${ }^{16}$ A. Vahid, A. D. Sans, and J. R. Elliott, Ind. Eng. Chem. Res. 47, 7955 (2008).

${ }^{17}$ F. S. Baskaya, N. H. Gray, Z. N. Gerek, and J. R. Elliott, Fluid Phase Equilib. 236, 42 (2005).

${ }^{18}$ A. M. Hassan, D. T. Vu, D. A. Bernard-Brunel, J. R. Elliott, D. J. Miller, and C. T. Lira, Ind. Eng. Chem. Res. 51, 3209 (2012).

${ }^{19}$ E. M. Curtis and C. K. Hall, J. Phys. Chem. B 117, 5019 (2013).

${ }^{20}$ H. D. Nguyen and C. K. Hall, Biophys. J. 87, 4122 (2004).

${ }^{21}$ M. C. dos Ramos, H. Docherty, F. J. Blas, and A. Galindo, Fluid Phase Equilib. 276, 116 (2009).

${ }^{22}$ M. N. Bannerman, R. Sargant, and L. Lue, J. Comput. Chem. 32, 3329 (2011).

${ }^{23}$ G. Chapela, L. E. Scriven, and H. T. Davis, J. Chem. Phys. 91, 4307 (1989).

${ }^{24}$ J. Torres-Arenas, L. A. Cervantes, A. L. Benavides, G. A. Chapela, and F. del Rio, J. Chem. Phys. 132, 034501 (2010).

${ }^{25}$ G. A. Chapela, F. del Rio, A. L. Denavides, and J. Alejandre, J. Chem. Phys. 133, 234107 (2010).

${ }^{26}$ S. Ucyigitler, M. C. Camurdan, and J. R. Elliott, Ind. Eng. Chem. Res. 51, 6219 (2012).

${ }^{27}$ G. A. Chapela, F. del Rio, and J. Alejandre, J. Chem. Phys. 138, 054507 (2013).

${ }^{28}$ P. Müller and T. Pöschel, Phys. Rev. E 87, 033301 (2013). 
${ }^{29}$ J. A. Barker and D. Henderson, J. Chem. Phys. 47, 4714 (1967).

${ }^{30}$ B. Smit, J. Chem. Phys. 96, 8639 (1992).

${ }^{31}$ N. B. Wilding, Phys. Rev. E 52, 602 (1995).

${ }^{32}$ G. Orkoulas and A. Z. Panagiotopoulos, J. Chem. Phys. 110, 1581 (1999).

${ }^{33}$ N. B. Wilding, Am. J. Phys. 69, 1147 (2001).

${ }^{34}$ M. N. Bannerman and L. Lue, J. Chem. Phys. 133, 124506 (2010).

${ }^{35} \mathrm{~K}$. Meier, Computer simulation and interpretation of the transport coefficients of the Lennard-Jones model fluid, Ph.D. thesis, Dept. Mech. Eng., Uni. Fed. Armed forces, Hamburg (2002).

${ }^{36}$ K. Meie, A. Laesecke, and S. Kabelac, J. Chem. Phys. 121, 3671 (2004).

${ }^{37}$ K. Meie, A. Laesecke, and S. Kabelac, J. Chem. Phys. 121, 9526 (2004).

${ }^{38}$ M. Bugel and G. Galliero, Chem. Phys. 352, 249 (2009).

${ }^{39}$ P. Valentini and T. E. Schwartzentruber, J. Comput. Phys. 228, 8766 (2009).

${ }^{40}$ S. Miller and S. Luding, J. Comput. Phys. 193, 306 (2004). 


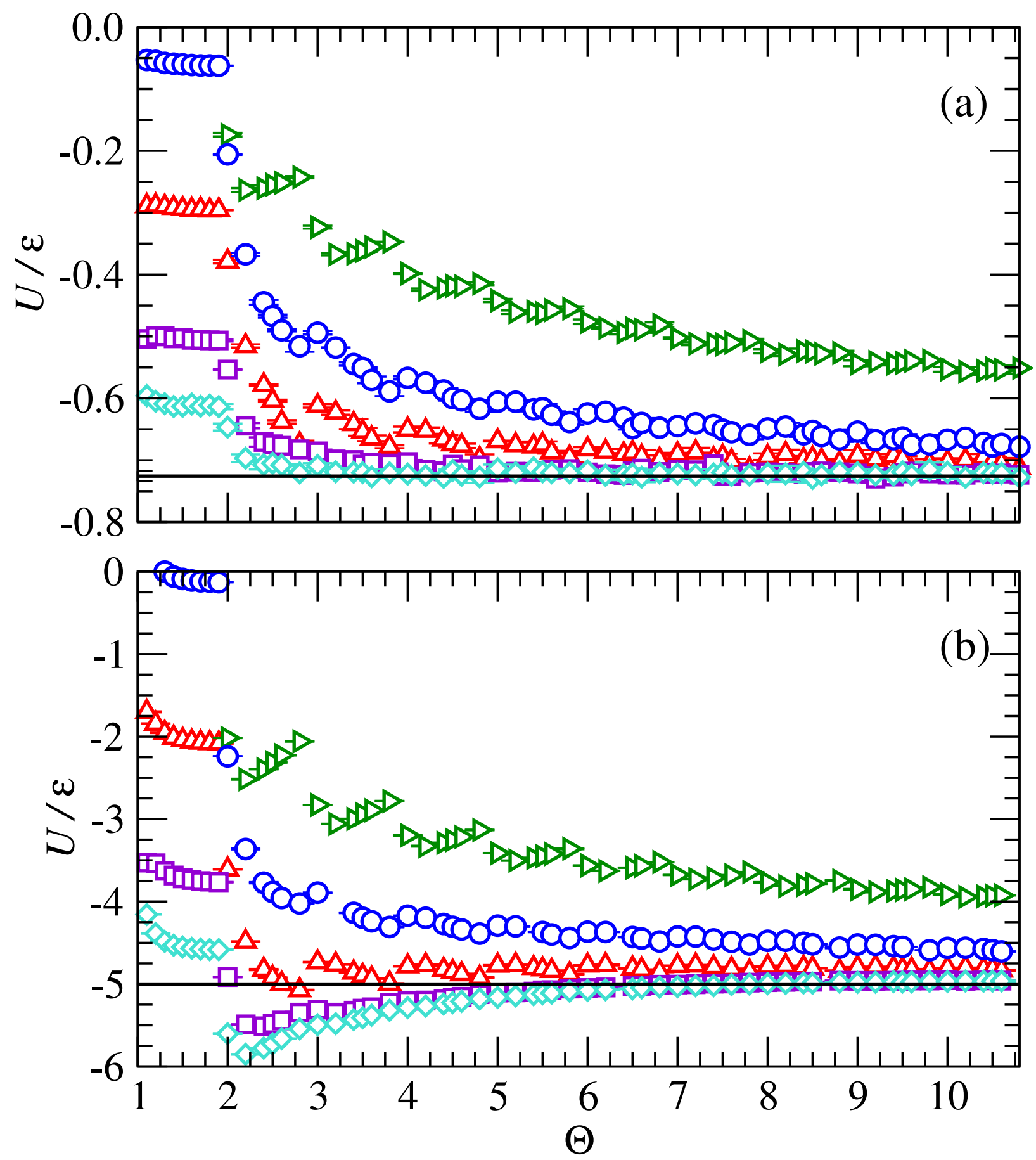

FIG. 4. A comparison of the stepped potential predictions for the internal energy $U$ for the system described in Fig. 3. 


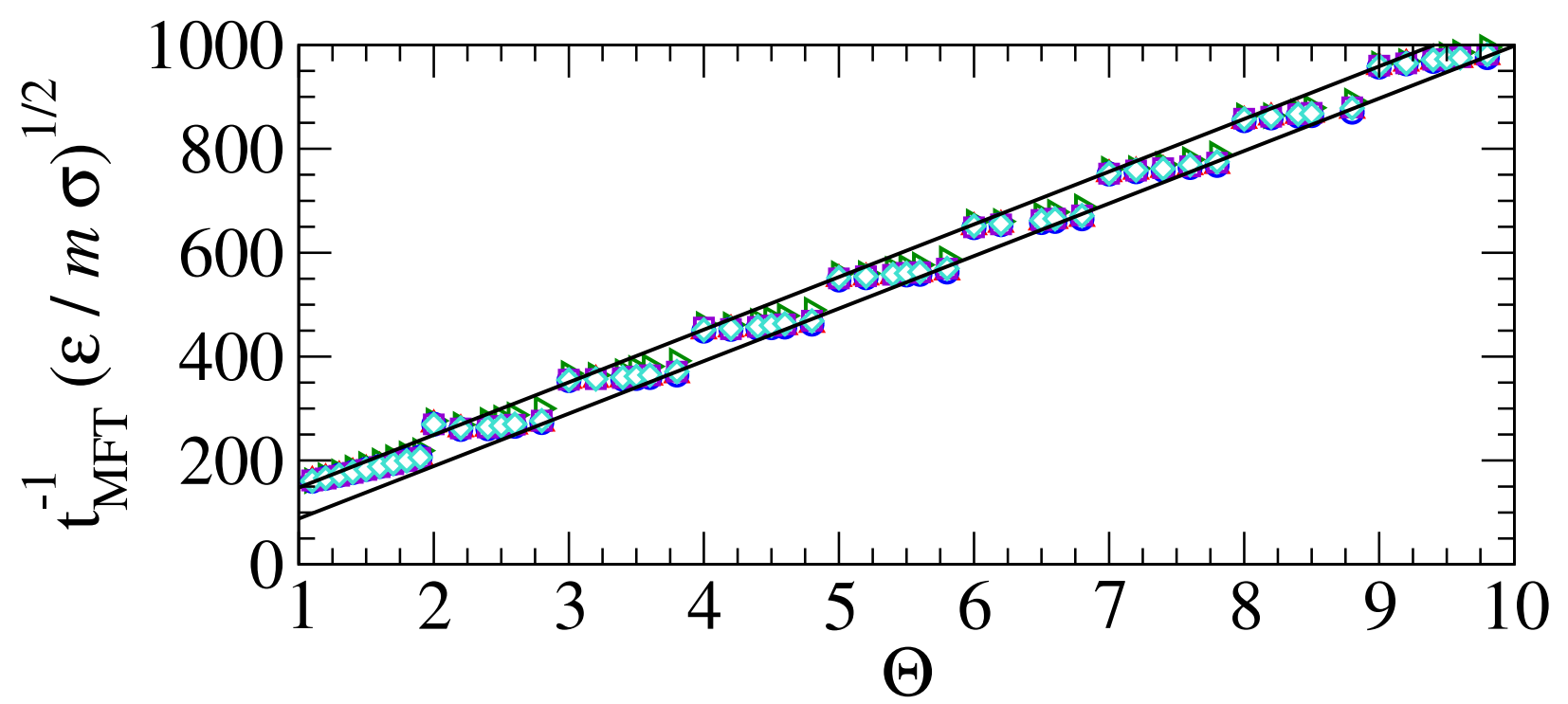

FIG. 5. The events per particle per unit of simulation time, given by $t_{M F T}^{-1}=2 N_{\text {events }} /\left(N t_{\text {sim }}\right)$ where $N_{\text {events }}$ is the number of events caused by a particle pair encountering a discontinuity during a simulation of duration $t_{\text {sim }}$, for various step placement algorithms at a temperature of $k_{B} T / \varepsilon=1.3$ and density of $\rho \sigma^{3}=0.85$. The symbols are described in Fig. 3 and the straight lines have been regressed to the virial data points for $\Theta>2$ with fractional parts of .0 and .8 .

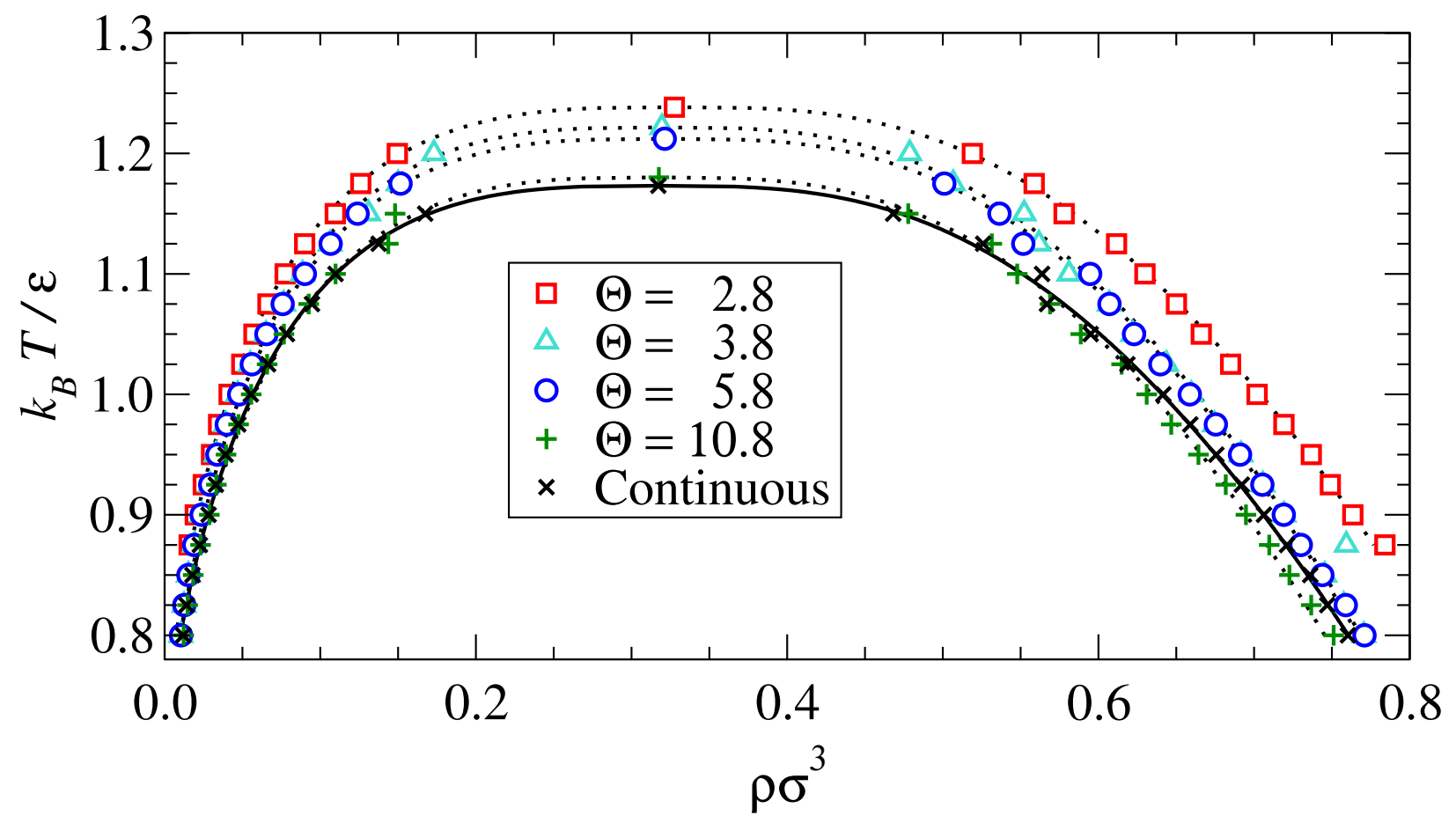

FIG. 6. Phase diagram of the $r_{\text {cutoff }}=3 \sigma$ Lennard-Jones fluid. Symbols denote MCMC results and the extrapolated critical point. Lines are the best fits of Eqs (12) and (13). 


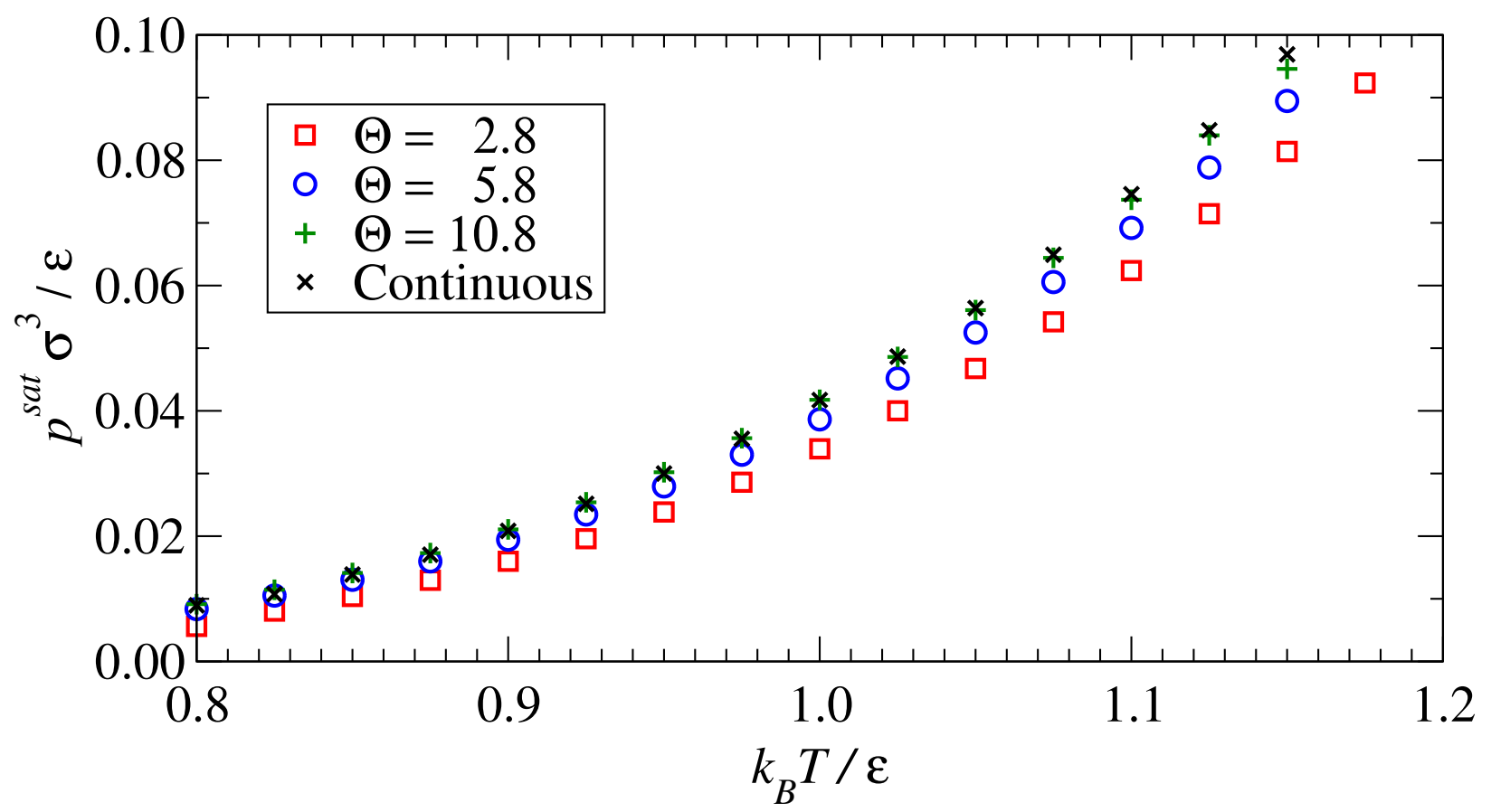

FIG. 7. Vapor pressures $p^{\text {sat }}$ of the $r_{\text {cutoff }}=3 \sigma$ stepped and continuous Lennard-Jones fluid. 

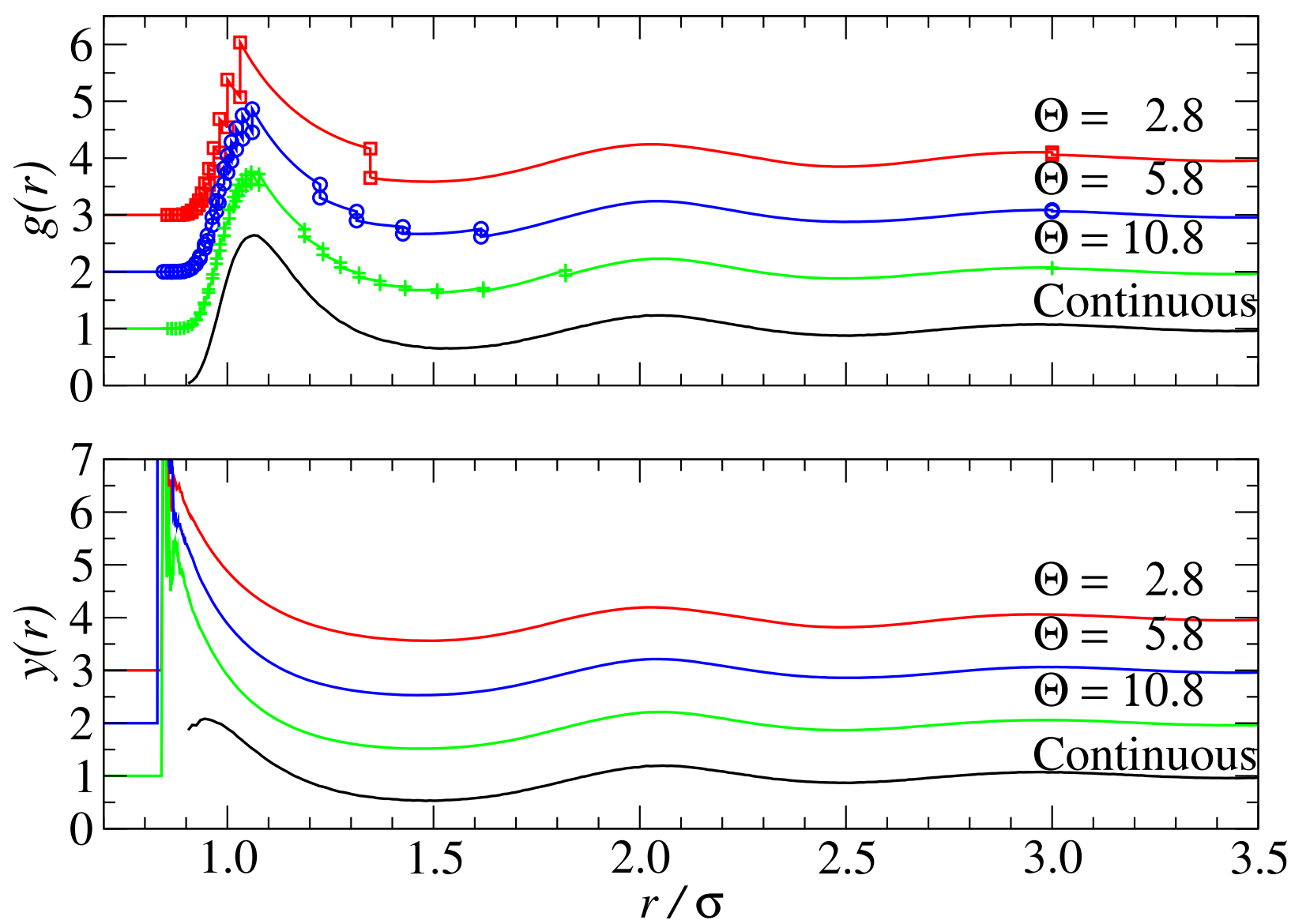

FIG. 8. The (a) radial distribution function, $g(r)$, and the (b) cavity correlation function, $y(r)$, for the stepped and continuous Lennard-Jones potential at $k_{B} T=1.3$ and $N / V=0.85$. Curves have been shifted to ease comparison and symbols denote values either side of the discontinuities as calculated from the event-rate formulas ${ }^{34}$. 

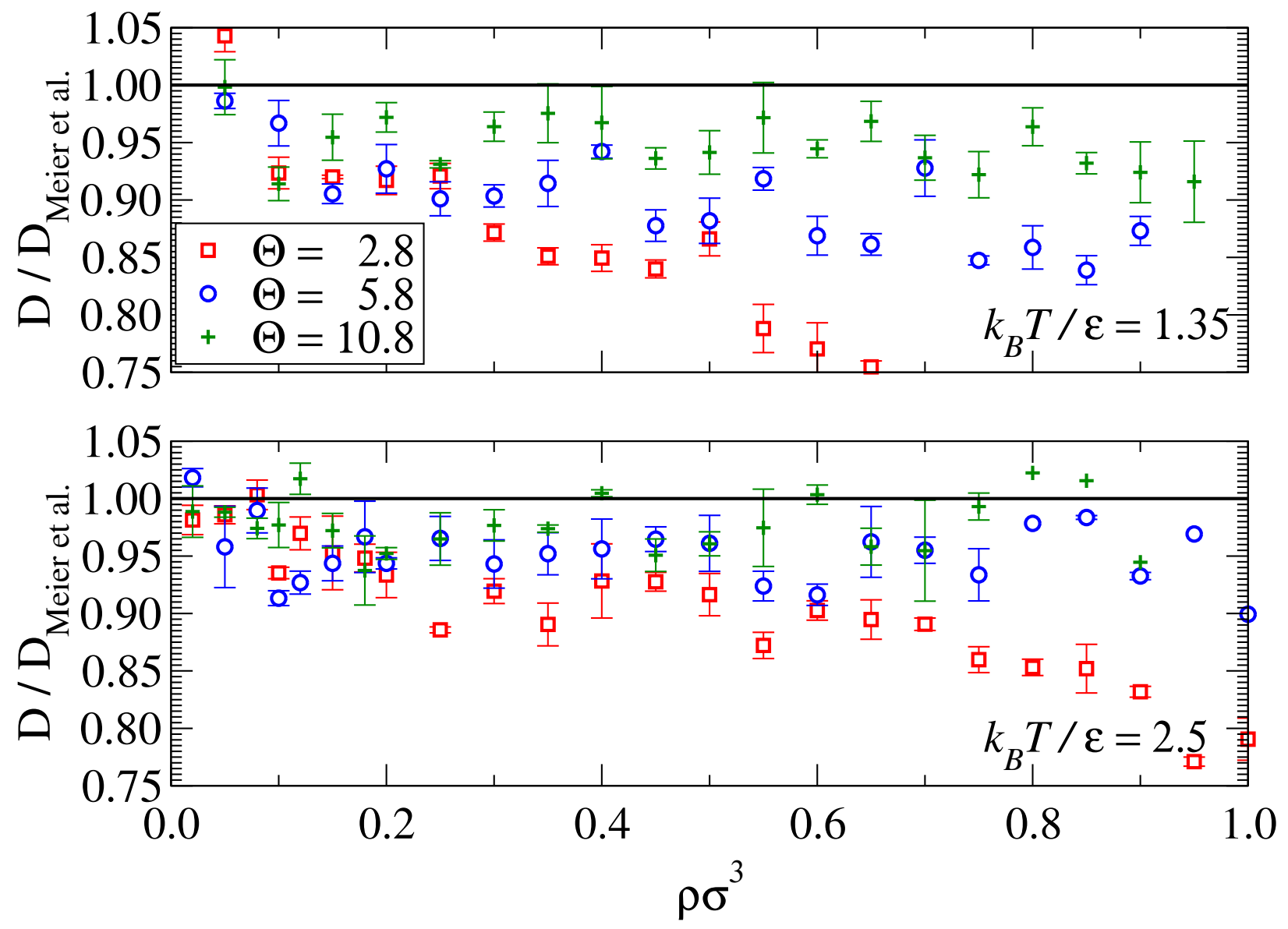

FIG. 9. Diffusion coefficients relative to the reference continuous-potential data for two isotherms over a range of densities. Reference data is taken from Meier et al. ${ }^{37}$. 

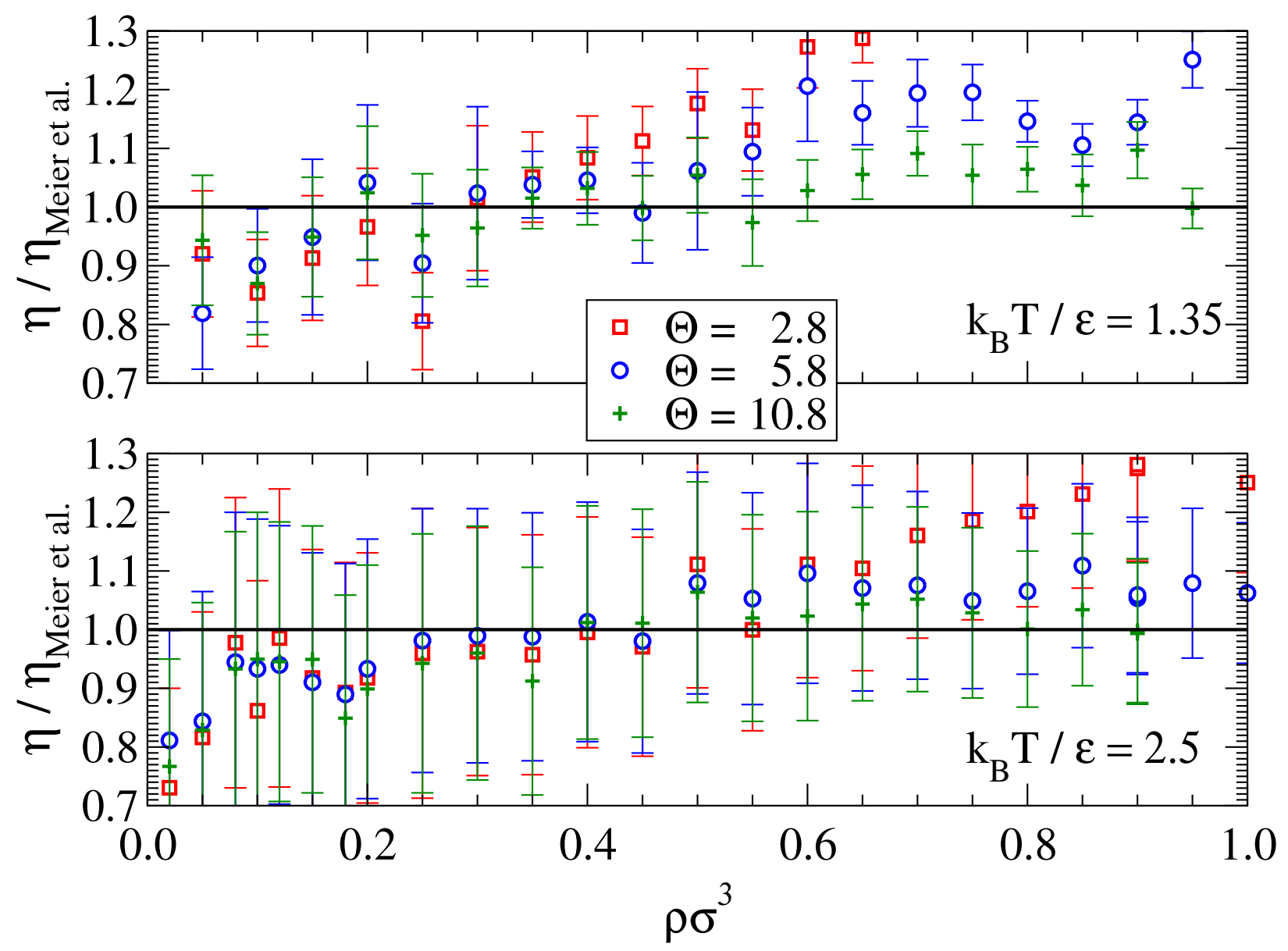

FIG. 10. Shear viscosity coefficients relative to the reference continuous-potential data for two isotherms over a range of densities. Reference data is taken from Meier et al. ${ }^{36}$. 

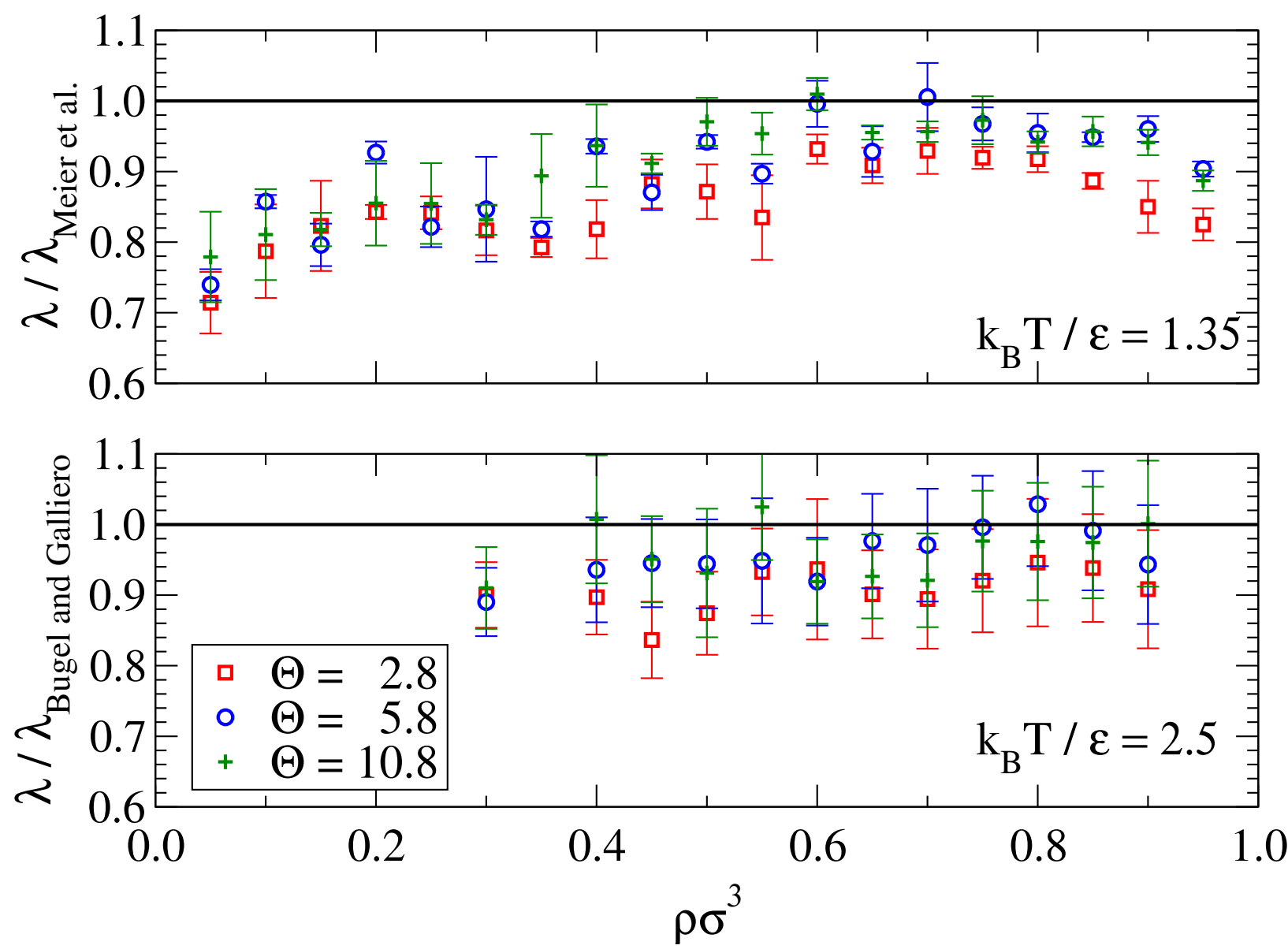

FIG. 11. Thermal conductivity relative to the reference continuous-potential data for two isotherms over a range of densities. Reference data is taken from Meier et al. ${ }^{35}$, and Bugel and Galliero ${ }^{38}$. 


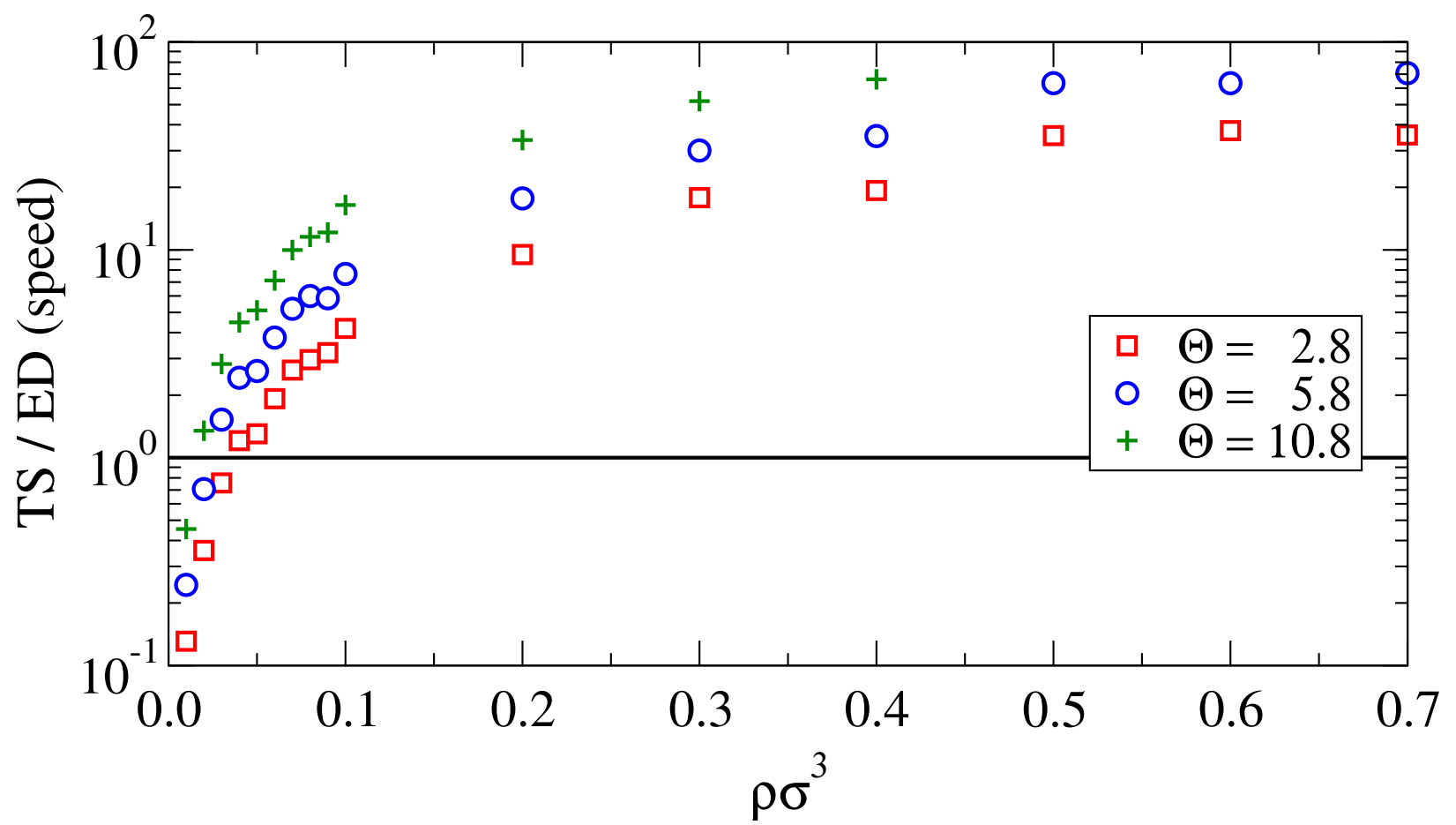

FIG. 12. The relative calculation-speed (simulation time processed per unit of CPU time) of time-stepping (TS) and event-driven (ED) molecular dynamics for a Lennard-Jones isotherm at $k_{B} T / \varepsilon=1.3$. Symbols correspond to different stepped approximations. 